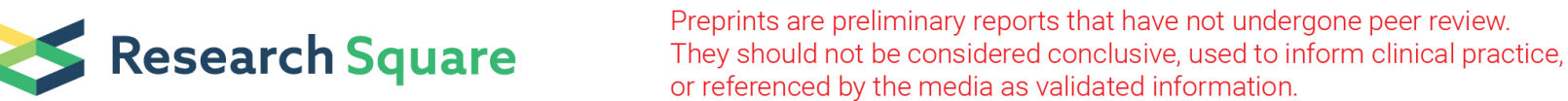

\section{Development and Pilot-testing of a Hepatitis C Reinfection Prevention Intervention for Patients in Treatment for Hepatitis C Infection}

\section{Adam Christopher Viera}

Yale University School of Public Health https://orcid.org/0000-0003-3248-0478

\section{Lauretta E. Grau}

Yale School of Public Health

Jeffrey D. Fisher

University of Connecticut Institute for Collaboration on Health, Intervention, and Policy

\section{Scott O. Farnum}

APT Foundation

Jeanette M. Tetrault

Yale School of Medicine

\section{Greg Scott}

DePaul University

Robert Heimer ( $\nabla$ robert.heimer@yale.edu )

Department of Epidemiology of Microbial Diseases, Yale University School of Public Health, New Haven, CT, USA https://orcid.org/0000-0003-0589-6697

\section{Research}

Keywords: hepatitis C virus (HCV), Information-Motivation-Behavioral Skills (IMB), COVID-19 pandemic

Posted Date: January 5th, 2021

DOI: https://doi.org/10.21203/rs.3.rs-139092/v1

License: (c) (1) This work is licensed under a Creative Commons Attribution 4.0 International License. Read Full License

Version of Record: A version of this preprint was published at Drug and Alcohol Dependence Reports on March 1st, 2022. See the published version at https://doi.org/10.1016/j.dadr.2022.100038. 


\section{Abstract}

Background: With annual rates of hepatitis $\mathrm{C}$ virus (HCV) reinfection among persons who inject drugs ranging from $5 \%$ to $22 \%$, the need for behavioral interventions to prevent reinfection following successful treatment is clear.

Methods: This report aims to describe the conception and development of an intervention to prevent HCV reinfection and present preliminary results from its pilot testing at an opioid treatment program offering on-site primary medical care, including treatment for HCV infection. We developed a two-session intervention combining a teachable moment followed by a session based on the Information-MotivationBehavioral Skills (IMB) Model to reinforce learning. The teachable moment occurs in less than ten minutes during the routine blood draw to measure viral load during HCV treatment. The reinforcing IMB session builds knowledge, motivation, and self-efficacy in practicing safer injection skills. Formative exploratory work for the intervention involved a literature review, planning meetings with implementation staff, and development of study materials. Intervention staff were trained and the intervention was pilot tested. Measured outcomes included feasibility and acceptability of the intervention to patients and implementers, study recruitment and retention, and preliminary changes in knowledge, motivation, selfefficacy, and HCV reinfection risk behaviors over the study time period.

Results: The study protocol and intervention content are both described, along with the preliminary results of implementation and psychosocial outcomes among 17 patients undergoing direct-acting antiviral treatment for HCV. Baseline data revealed gaps in HCV prevention knowledge that could lead to reinfection. We also report lessons learned related to implementation of such an intervention with this population in integrated care settings. After some minor adjustments, we found high levels of feasibility and acceptability for the intervention at the implementing organization. Intervention implementation was interrupted due to COVID-19 restrictions.

Conclusions: It is possible to implement an intervention in an opioid treatment program to improve HCV prevention knowledge with the potential to prevent reinfection. Intervention staff must be attentive to participant needs regarding time and monetary constraints to maximize acceptability. Given the impact of the COVID-19 pandemic, future research should explore the possibility of offering the intervention via phone or video chat.

\section{Background}

Hepatitis $\mathrm{C}$ virus (HCV) infection is a major health concern in the U.S., with an estimated 2.5 million Americans living with HCV (1) and rates of acute HCV infection increasing as a result of the opioid epidemic (2). Direct-acting antiviral (DAA) medications demonstrate almost universal, pangenomic success in resolving HCV infection (3-5); however, their impact on prevalence of active HCV infection is negatively impacted by HCV reinfection $(6,7)$. Expanding access to and completion of HCV treatment among persons who inject drugs (PWID) has the potential to reduce community viral load and prevent 
future HCV infection (8). Rates of HCV reinfection among PWID have been observed to range from 5$22 \%$ annually $(9-11)$, suggesting that preventing HCV reinfection remains an overlooked step in the HCV care continuum (12).

Behavioral interventions can reduce behaviors that risk HCV infection among PWID. Those with demonstrated success change the risk environment for PWID and are especially effective when implemented in combination with substance use treatment $(13,14)$. Researchers have posited that HCV treatment can serve as a critical period for intervention to prevent future HCV reinfection (11). As such, we designed a behavioral intervention for individuals starting treatment for HCV infection to build knowledge, motivation, and self-efficacy to engage in safer injection practices and avoid HCV reinfection.

Formative work by several of the co-authors was critical in identifying the need for intervention in this population. This work highlighted the need for education and awareness around the risk of HCV infection through sharing of syringes and other injection equipment $(15,16)$. This work also identified the durability of infectious HCV in injection equipment weeks and even months after contamination $(17,18)$. Formative work around a brief intervention to prevent HCV infection highlighted the importance of behavioral interventions that move beyond passive education while reinforcing risk reduction messaging (19); selfefficacy was identified as an especially salient factor to address among PWID who do not attend syringe service programs (20). These findings were key in identifying the Information-Motivation-Behavioral Skills (IMB) Model as a potential framework for designing and developing an intervention. The IMB Model demonstrates that behaviors and behavior change are related to knowledge of the problem and strategies to overcome it, motivation to engage in less risky behavior, and belief that one has the skills needed to engage in the behavior (21). The IMB Model has been used previously in the design of multiple behavioral interventions (22) and is an appropriate model to employ based on the determinants identified in the formative work for this intervention.

The research team used the IMB Model to inform the design of the intervention. In this article, we report findings from a pilot intervention curtailed as a result of the COVID-19 pandemic. We believe that the data presented, though limited, provide a guide for future efforts to curtail HCV reinfection among PWID.

\section{Methods}

The intervention was developed and piloted through a collaboration between the research team and an implementation team at an opioid treatment program (OTP) where patients were receiving DAA treatment. The APT Foundation in New Haven is among the largest OTPs in Connecticut, with over 5,000 patients. It provides primary medical care and, since 2015 , has provided onsite HCV treatment using DAAs. Of the patients initiating treatment in this onsite model, $98 \%$ completed DAA treatment despite ongoing drug use reported by one quarter of patients in the treatment cohort (23). Retention in treatment for opioid use disorder (OUD) was greater for those whose HCV infection was treated in this model compared to those who chose not to pursue DAA treatment onsite (24). This high rate of treatment 
completion and retention in OUD treatment made the APT Foundation the ideal location to pilot test the proposed behavioral intervention.

\section{Implementation team}

In addition to the research team, a four-member implementation team from the APT Foundation was trained to conduct all aspects of the intervention. The implementation team member tasked with conducting the group session was a Licensed Drug and Alcohol Counselor.

\section{Intervention development process}

To guide the process of developing the pilot intervention, we used the Stage Model of Behavioral Therapies Research (25). According to this model, the process of developing and evaluating a behavioral intervention falls into two stages. Stage 1 focuses on the development process and is broken into two substages: the intervention is developed and manualized and intervention staff are trained in stage 1a and the intervention is pilot tested in stage $1 \mathrm{~b}$. Stage 2 involves testing the efficacy of the intervention. In this article, we describe the Stage 1 process.

\section{Intervention structure}

The intervention itself was conceived as a two-session process to be delivered during the course of DAA treatment at the APT Foundation. The brief, initial session used treatment-related phlebotomy to provide instruction on the essentials of safe injection followed by a longer, second session informed by the principles of the IMB Model to reinforce the visual learning experience. The first session uses principles of vicarious learning to improve knowledge of safer injection practices (26) while the second session was designed according to elements of social cognitive theory more broadly (27).

\section{First Session: The 10-Minute Intervention (10-MI)}

Studies designed to provide hepatitis B vaccination to active drug users provided the impetus for this brief intervention $(28,29)$. Ethnographic observations conducted in a non-medical settings confirmed earlier quantitative studies that revealed that study participants were often unaware of the basics of injection hygiene (19); however, they were amenable to learning more when safer injection education was offered in the context of blood draws for hepatitis testing. Treating the first session as a "teachable moment," content for the first session was limited to what could be delivered during a 10-minute or shorter blood draw, with the intervention content to be delivered by a trained, non-clinical staff person narrating the protective actions that the phlebotomist took during a routine blood draw. The session covered five key steps: (1) preparing a clean space for injection, (2) finding a vein, (3) getting the skin ready for injection, (4) registering the needle in the vein, and (5) cleaning up after injection.

\section{Second Session: The IMB Intervention}

In order to build on the existing evidence base surrounding behavioral interventions targeting components of the IMB Model (i.e., knowledge, motivation, behavioral skills, self-efficacy), the research team 
conducted a review of the literature since 2000 that specifically focused on peer-reviewed articles about interventions to reduce HCV infection and reinfection risk behaviors. We extracted information about intervention components, relevant measures, and observed outcomes. Consistent with the IMB Model, the research team agreed that the second session would be a group session and would focus on reinforcing information presented in the first session, correcting misinformation, and building motivation and selfefficacy around safer injection practices.

The second session was designed to last an hour, based on the structure of other group sessions offered by the APT Foundation. The IMB Intervention had four sequential components. The first provided an interactive review of the information presented during the 10-MI. The second brainstormed reasons and social supports that motivate safer injection moving forward. The third built self-efficacy around safer injection by discussing and demonstrating key elements of syringe cleaning, drug splitting, and reducing exposure to blood on surfaces and injection equipment. The fourth provided harm reduction resources and referrals. The IMB Intervention incorporated interactive elements to increase engagement and ensure more effective transfer of information and skills.

\section{Development of intervention materials}

Based on this intervention structure and content, a plain-language script was developed for the first intervention session, highlighting key points to be covered and including supplemental information for facilitators to use when answering any patient questions. Some of the intervention handouts were adapted from existing harm reduction resources developed by the Chicago Recovery Alliance (30), CATIE (Canadian AIDS Treatment Information Exchange) (31), and the authors of the SHIELD (Self-Help In Eliminating Life-threatening Diseases) intervention, a peer-based intervention focused on adoption of safer sex and drug use behaviors among persons who use drugs (32). Materials were reviewed by the research team and then presented to the implementation team for their review and commentary. After all feedback was incorporated, the materials were finalized and approved by the Yale University Institutional Review Board prior to use in the pilot project.

\section{Study protocol to test intervention}

In keeping with the Stage Model of Behavioral Therapies Research, we conducted a Stage 1b pilot in order to test the feasibility of delivering the intervention and assess its effect on immediate and intermediate outcomes related to safer injection.

The research team decided on a pre-/post-test design, where measures of outcome variables were collected after each intervention session and again approximately 12 weeks and 24 weeks after HCV treatment completion, as depicted in Fig. 1. No control group was selected for this pilot study. Our initial goal was to recruit 75 participants.

\section{Participant recruitment and data collection schedule}


All participants were patients at the OTP who were planning to start treatment for HCV infection. Receipt of medications for OUD was not a criterion for study inclusion. In addition to the above, participants were eligible if they: (1) were over 18 years of age, (2) spoke English, and (3) provided informed consent. Participants were initially offered no monetary reimbursement for participation in the study, though participation in the second session satisfied group counseling requirements for those participants receiving OUD treatment. Later in the study, participants were all offered a monetary reimbursement totaling \$60 provided on a stepped scale: \$10 for completion of the baseline survey, \$10 for completion of the survey after the 10-MI, $\$ 10$ for completion of the first follow-up survey, and $\$ 30$ for completion of the second and final follow-up survey. All participants who had consented to participate prior to the additional monetary reimbursement were contacted and reconsented to provide them with the same reimbursement retroactively. Rationale for the change in reimbursement is provided in the Results section.

Patients starting treatment for their HCV infection were approached by members of the implementation team, who assessed the patient's interest in the study and conducted the informed consent process. Those who consented then completed the baseline assessment, which collected information on participant demographic characteristics, history of injection practices, and knowledge of syringe access and HCV infection.

After enrollment into the study, the 10-MI was delivered at approximately the fourth week of HCV treatment during a blood draw that is part of regular care to assess the impact of DAA treatment on HCV viral load. Afterwards, participants completed a brief assessment of safer injection knowledge, motivation, intention, self-efficacy, and behaviors (hereafter referred to as the safer injection assessment). Approximately two weeks later, participants were invited to attend the IMB Intervention, after which they repeated the safer injection assessment and completed a satisfaction survey. Upon completion of HCV treatment, participants again completed the safer injection assessment two more times. The timing of these follow-up assessments coincided with post-treatment viral load testing and therefore varied based on treatment regimen. Participants completed the first assessment around 12 weeks after treatment completion and the second assessment around 24 weeks after treatment completion. This schematic is depicted in Fig. 1.

\section{Process Measures}

We sought to identify the elements of the intervention that could be feasibly, acceptably, and reliably implemented. Feasibility data were collected pertaining to recruiting, engaging, retaining, and collecting longitudinal data from participants at each study visit. We assessed recruitment feasibility by comparing the number of individuals starting HCV treatment to the number who agreed to participate in the study. We also took note of common reasons given for not agreeing to participate. We assessed the feasibility of engagement in the intervention by charting the extent to which participants took part in intervention activities within the allotted time frame. We assessed retention feasibility by comparing the total number of participants who enrolled in the study to the number who completed all relevant benchmarks. Finally, we assessed the feasibility of data collection, as it was conducted, by examining the percentage of complete responses for all intervention data collection instruments. 
Acceptability of the intervention was assessed by providing participants with a survey to rate their satisfaction with the intervention components and to comment on the elements of the intervention that they appreciated and those they thought should be changed. Participants completed the satisfaction survey after completing the IMB session.

For this study, we did not formally assess fidelity of implementation staff to deliver the intervention according to the written protocol. We did convene regular meetings with the implementation team to assess their experiences and challenges they felt prevented delivering the intervention according to the protocol.

\section{Outcome measures}

In keeping with the pilot setting and the IMB Model framework, a safer injection assessment was developed to collect data on a number of immediate, intermediate, and long-term outcomes and sought to capture changes over time in safer injection knowledge, motivation, self-efficacy, intention, and behavior.

In order to assess safer injection knowledge, we adapted questions from the results of previous studies with "True", "False", and "Don't Know" as response options (20,33). Participants were directed to select the "Don't Know" option only in those cases where they could not hazard a guess as to the correct response. This battery of 10 questions covered the content of both intervention sessions.

To assess motivation to engage in safer injection in the future, we used an adapted version of the Treatment Self-Regulation Questionnaire from the larger Health-care, Self-Determination Theory Questionnaire $(34,35)$. We used items developed by Khatmi and colleagues $(36)$ to inform our modification of the questionnaire items. The finalized scale included eight items, with a five-point Likert scale of response options: "Strongly Disagree", "Disagree”, "Neither Agree Nor Disagree", "Agree”, and "Strongly Agree". In developing the scale, we were careful to ensure a balance of items related to intrinsic and extrinsic motivations to engage in safer injection.

The assessment of self-efficacy to practice safer injection steps adapted a version of the Health-care, Self-Determination Theory Questionnaire, modifying items from the Perceived Competence Scale (35). As before, items developed by Khatmi et al. (36) inspired our development of items for this scale. Items assessing self-efficacy included performing steps of safer injection (e.g., setting up injection equipment, finding a vein) and refusing to share syringes and other injection equipment. Based on work by Marotta and colleagues (37) and Latka and colleagues (38), the final instrument had 10 items and the same fivepoint Likert scale as the safer injection motivation questions.

We assessed engagement in injection drug use in the past three months. If participants indicated that they injected in the past three months, they were asked about the frequency of engaging in specific injection behaviors such as drug splitting, syringe cleaning, and syringe sharing. We asked a total of six questions about safer injection behaviors, with a five-point Likert scale of response options: "Never", "Almost Never", Half the Time", "Almost Always", and "Always". We also aimed to assess the participants' 
intentions to practice safer injection if they were to inject in the future. We developed a behavioral intention scale that asked participants to rate their likelihood of future engagement in the same six injection behaviors assessed in the behavioral scale, using the same Likert scale and response options.

\section{Results}

We recruited a total of 17 participants into the pilot study over a ten-month period (from May 2019 through February 2020). Recruitment was halted when the SARS-CoV-2 pandemic in Connecticut resulted in shelter in place and quarantine orders that curtailed HCV treatment offered by the OTP and prevented further intervention and data collection activities.

\section{Description of Study Sample}

Table 1 summarizes the pilot study participants' characteristics. Participants were mostly male (70.6\%), reporting an average age of 41.9 at baseline. Participants reported a high level of education, with $41.2 \%$ reporting some level of college or technical school. Most participants were unemployed (58.8\%) and, of those who were employed, most were employed part-time (85.7\%). All participants had health insurance, either Medicaid (88.2\%) or Medicare (23.5\%), with two participants reporting that they had both. 
Table 1

Participant Characteristics $(\mathrm{N}=17)$

\begin{tabular}{|c|c|}
\hline & $\mathrm{N}(\%)$ or Mean (SD) \\
\hline Gender & $5(70.6 \%)$ \\
\hline Female & $12(29.4 \%)$ \\
\hline \multicolumn{2}{|l|}{ Male } \\
\hline Age & $41.9(13.8)$ \\
\hline Education & $1(5.9 \%)$ \\
\hline 8th grade or less & $3(17.6 \%)$ \\
\hline Some high school & $6(35.3 \%)$ \\
\hline High school graduate/GED & $7(41.2 \%)$ \\
\hline \multicolumn{2}{|l|}{ Some college/technical school } \\
\hline Employment & $10(58.8 \%)$ \\
\hline Not employed & $1(5.9 \%)$ \\
\hline Full-time & $6(35.3 \%)$ \\
\hline \multicolumn{2}{|l|}{ Part-time } \\
\hline Insurance & $13(76.5 \%)$ \\
\hline Medicaid & $2(11.8 \%)$ \\
\hline Medicare & $2(11.8 \%)$ \\
\hline \multicolumn{2}{|l|}{ Medicaid \& Medicare } \\
\hline Arrested & $15(93.8 \%)$ \\
\hline Felony Conviction & $8(50.0 \%)$ \\
\hline Probation or Parole & $5(29.4 \%)$ \\
\hline Awaiting Charges & $3(17.6 \%)$ \\
\hline Past Month Alcohol Use & $3(18.5 \%)$ \\
\hline Past Month Cocaine Use & $4(25.0 \%)$ \\
\hline Past Month Methamphetamine Use & $1(5.9 \%)$ \\
\hline Past Month Opioid Use & $0(0.0 \%)$ \\
\hline Past Month Street Fentanyl Use & $3(18.8 \%)$ \\
\hline Past Month Injection & $3(17.6 \%)$ \\
\hline
\end{tabular}


Almost all participants had some involvement with the criminal justice system, with all but one (93.8\%) having ever been arrested. Half had been convicted of a felony, nearly a third $(29.4 \%)$ were on parole or probation, and $17.6 \%$ were currently awaiting trial or some other court appearance.

All participants reported a history of injection drug use. Only three (17.6\%) reported injecting drugs in the month before enrollment into the study, with most of these participants $(66.7 \%)$ reporting access to sterile syringes. In the past month, three participants (18.8\%) reported alcohol use, four (25.0\%) reported cocaine use, one $(5.9 \%)$ reported methamphetamine use, and three $(18.8 \%)$ reported use of street fentanyl. No participants reported the use of other opioids in the month prior.

\section{Baseline Syringe Access and HCV Knowledge}

At baseline, we also assessed participant knowledge of viral hepatitis infection as well as syringe access. Results are presented in Table 2. All participants knew that there is a vaccine to prevent hepatitis $B$ infection, but little more than half $(58.8 \%)$ knew that there was no vaccine for hepatitis $\mathrm{C}$. Nearly all participants knew that you cannot tell someone is infected with HCV by how they look (94.1\%) and that reinfection with hepatitis $C$ is possible even after being cured (94.1\%). A majority of participants knew that HCV infection can cause liver cancer (88.2\%), that drinking alcohol can make HCV infection worse (76.5\%), and that you cannot get HCV from contaminated food (70.6\%). Major knowledge gaps were found in knowing that most people with hepatitis B virus (HBV) infection recover from it $(23.5 \%)$ or that protecting skin after injection can help prevent HCV infection (11.8\%). Regarding syringe access, few participants knew that it is legal to carry syringes in Connecticut (17.6\%) and little more than half (58.8\%) knew that it is legal to buy syringes from a pharmacy without a prescription. 
Table 2

Baseline Knowledge of Hepatitis C and Syringe Access ( $n=17)$

\begin{tabular}{|ll|}
\hline Question & Number (\%) Correct \\
\hline HCV can cause liver cancer & $15(88.2 \%)$ \\
\hline HIV is easier to spread than HCV & $8(47.1 \%)$ \\
\hline You can prevent HCV spread by covering the skin where you just injected & $2(11.8 \%)$ \\
\hline You can tell someone is infected with HCV by the way they look & $16(94.1 \%)$ \\
\hline Drinking alcohol makes HCV worse & $13(76.5 \%)$ \\
\hline There is a vaccine to prevent HCV infection & $10(58.8 \%)$ \\
\hline There is a vaccine to prevent HBV infection & $17(100.0 \%)$ \\
\hline Most people who get infected with HCV totally recover from it & $9(52.9 \%)$ \\
\hline Most people who get infected with HBV totally recover from it & $4(23.5 \%)$ \\
\hline You can get HCV from contaminated food & $12(70.6 \%)$ \\
\hline HCV is easily spread by both sex and used needles & $2(11.8 \%)$ \\
\hline Once you have been cured of HCV, it is impossible to get reinfected & $16(94.1 \%)$ \\
\hline It is legal to buy syringes without a prescription in pharmacies in CT & $10(58.8 \%)$ \\
\hline It is legal in CT to carry used syringes & $3(17.6 \%)$ \\
\hline It is legal in CT to carry injection gear other than syringes* & $3(50.0 \%)$ \\
\hline${ }^{*}$ Question was only asked of 6 participants before the form changed & \\
\hline
\end{tabular}

\section{Implementation outcomes}

\section{Feasibility of recruitment and enrollment}

During the study recruitment period, a total of 83 unduplicated individuals started treatment for HCV infection at the APT Foundation. Of these, 17 (20.5\%) were enrolled into the study. Common reasons for declining participation were the lack of financial incentive and the time commitment involved in completing assessments. In response, the study team began to offer a graduated incentive for participation in the study, which resulted in an increased rate of recruitment into the study. In addition to the abovementioned challenges, there was a lag in recruitment efforts due to staff turnover. After training an intake nurse to share information about the study and enroll participants, recruitment increased considerably.

Feasibility of retention 
Of the 17 individuals recruited into the study, three (17.6\%) completed both intervention sessions. Another five (29.4\%) participants completed the first session but did not complete the second session. It is important to note that five $(29.4 \%)$ participants were recruited in the month prior to the termination of study activities due to COVID-19. Two of these participants had completed the first intervention session while the remaining three had not completed any intervention sessions.

\section{Feasibility of intervention delivery}

Meetings with the implementation team were useful in identifying areas where the intervention needed amending to be more successfully implemented in the pilot setting. Based on these meetings, the second session of the intervention was changed from a group session to a one-on-one session due to difficulties in coordinating participants' schedules and on-site logistics. The implementation team was able to use the same intervention materials and curriculum when facilitating the one-on-one sessions. Following this change, intervention delivery was reported as more feasible by the implementation team.

\section{Feasibility of data collection}

Complete data were collected from all participants at baseline. The main challenge in data collection at baseline was the opposite of what was anticipated; participants often completed sections that they should have skipped based on previous responses.

Data collection was more of a challenge in completing the safer injection assessments. Completion rates for this instrument ranged from $24.4-100 \%$ (mean completion rate: $82.9 \%$ ). After observing low rates of complete data in the safer injection assessments, the implementation team was provided additional training and practice in administering the assessments. As a result, data completion was lower in the first six months of the pilot compared to the final three months (66.3\% vs. $99.6 \%)$.

\section{Participant acceptability}

Only three participants completed the intervention, and two completed the satisfaction survey. Based on these surveys, participants rated both the intervention content and facilitators positively. Participants provided written comments about the intervention. One participant shared that they wish the intervention had been delivered in a "group setting". The other participants commented that they wanted the intervention to have more "physical instruction".

\section{Intervention outcomes}

Although not enough participants completed the safer injection assessment to draw any meaningful conclusions about interventions outcomes, the existing data show some trends. Knowledge of safer injection practices steadily increased for all participants who completed the assessment at multiple time points. Increases in safer injection motivation, self-efficacy, and intention were less consistent.

\section{Discussion}


The purpose of this study was to develop and pilot test a behavioral intervention to reduce HCV reinfection among individuals completing HCV treatment. Recruitment efforts and follow-up visits were discontinued after February 2020 due to the COVID-related restrictions that interrupted the study in the midst of recruitment for its first arm; as such, we were unable to reach the intended recruitment total of 75 participants.

Initial challenges in recruitment included the lack of a monetary incentive as well as interruptions in treatment initiation due to turnover of medical personnel at the implementing organization. After offering a small incentive for participation and training the intake nurse at the implementing organization, we experienced a considerable uptick in recruitment. Unfortunately, this came just prior to state-mandated and organization-instituted closure of in-person treatment service provision.

Although participants were fairly knowledgeable about some aspects of viral hepatitis prevention at baseline, most were ill-informed about actual transmission risks relative to the processes of injecting drugs, which is the most likely route of HCV infection and reinfection. This lack of knowledge is consistent with previous studies, and unchanged over two decades $(15,33)$, demonstrating the need for a highly targeted intervention of the kind we have developed.

Implementation team members reported high feasibility and acceptability of intervention activities, and the limited data available suggest high levels of satisfaction with intervention components. An unintended benefit of the intervention was that staff training resulted in higher levels of HCV knowledge among implementation team members. The main logistical challenge to feasibility of implementing the intervention was the difficulty of convening the group sessions, even though the OTP counted attendance at the session toward the counseling requirement mandated by federal regulations governing OTPs. Once the protocol was amended to allow the IMB session to be administered one-on-one, implementation of the intervention proceeded without any further issues. Hence, careful attention must be paid to balance the resource costs of convening groups with the intervention benefits, as described by at least one participant. Payments to compensate participants for coming at a specified time might permit a return to group sessions.

Assessing the feasibility of data collection was critical for consideration in planning for a future clinical trial of the intervention. Baseline data completion was high. Completion of the safer injection survey increased to nearly $100 \%$ after providing the implementation team with additional training and practice in completing and checking data collection instruments prior to the end of the session. Data collection could have further been improved throughout the pilot period by creating a digitally automated version that can be administered either by implementation staff or by the participant themselves; having a forced skip pattern would have prevented participants from answering unnecessary questions.

The scope of our findings is limited due to the interruption of the pilot by the COVID-19 response. As a result, we were unable to obtain all of the data on participant acceptability and outcomes following the intervention. While the preliminary data on feasibility and acceptability yielded important insights, we plan to conduct a larger intervention trial to better assess intervention outcomes. 


\section{Conclusions}

This formative work demonstrates the feasibility of implementing a behavioral intervention to prevent $\mathrm{HCV}$ reinfection in tandem with delivering DAA treatment for HCV infection. Baseline data show that participants report low levels of knowledge around safer injection practices, indicating that this group is in need of an intervention of this kind, especially as the available data suggest that the intervention could be successful in increasing safer injection knowledge over time. Future work will focus on evaluating the effect of the intervention on changes in knowledge, motivation, self-efficacy, intention, and behavior.

\section{Declarations}

\section{Ethics approval and consent to participate}

We sought approval to conduct this project from the Yale University Institutional Review Board. All participants provided informed consent prior to participation in the study.

\section{Consent for publication}

Not applicable.

\section{Availability of data and material}

All intervention materials and data will be made available by the corresponding author upon request.

\section{Competing interests}

This work was made possible through funding from Gilead Sciences, Inc.

\section{Funding}

This work was made possible through funding from Gilead Sciences, Inc. The funder was not involved in study design, intervention development, or data collection, analysis, and interpretation. The funder did review the manuscript prior to submission, but did not provide substantive feedback.

\section{Authors' contributions}

$\mathrm{RH}, \mathrm{LEG}, \mathrm{JDF}$, and GS conceived and planned the project. AV developed the intervention as described in collaboration with LG and JDF. SOF and JMT were part of the implementation team and supported the development and implementation of the intervention as described. AV wrote the manuscript with feedback and revision support from all authors.

\section{Acknowledgements}

Immense thanks to the APT Foundation team members who were instrumental in the development and implementation of the pilot intervention: Edin Bajraktarevic, Brian Bonilla, Heather Knox, and Dana Proto. 
Special thanks to our pilot participants.

\section{References}

1. Hofmeister MG, Rosenthal EM, Barker LK, Rosenberg ES, Barranco MA, Hall EW, et al. Estimating prevalence of hepatitis C virus infection in the United States, 2013-2016. Hepatology (Baltimore, Md). 2019;69(3):1020-31.

2. Zibbell JE, Asher AK, Patel RC, Kupronis B, Iqbal K, Ward JW, et al. Increases in acute hepatitis C virus infection related to a growing opioid epidemic and associated injection drug use, United States, 2004 to 2014. American journal of public health. 2018;108(2):175-81.

3. Falade-Nwulia O, Suarez-Cuervo C, Nelson DR, Fried MW, Segal JB, Sulkowski MS. Oral direct-acting agent therapy for hepatitis $C$ virus infection: a systematic review. Annals of internal medicine. 2017;166(9):637-48.

4. Graf C, Mücke MM, Dultz G, Peiffer K-H, Kubesch A, Ingiliz P, et al. Efficacy of direct-acting antivirals for chronic hepatitis $C$ virus infection in people who inject drugs or receive opioid substitution therapy: a systematic review and meta-analysis. Clinical Infectious Diseases. 2019;70(11):2355-65.

5. Alavian SM, Dolatimehr F, Sharafi H, Safi-Abadi M, Rezaee-Zavareh MS, Bayatpour ME, et al. Treatment of HCV infection with direct-acting antiviral agents in patients with HIV/HCV co-infection: a systematic review. Hepatitis monthly. 2018;18(12).

6. Midgard H, Weir A, Palmateer N, Lo Re V, 3rd, Pineda JA, Macias J, et al. HCV epidemiology in highrisk groups and the risk of reinfection. Journal of hepatology. 2016;65(1 Suppl):S33-s45.

7. Hellard M, McBryde E, Sacks Davis R, Rolls DA, Higgs P, Aitken C, et al. Hepatitis C transmission and treatment as prevention - The role of the injecting network. International Journal of Drug Policy. 2015;26(10):958-62.

8. Harris $\mathrm{M}$, Albers $\mathrm{E}$, Swan T. The promise of treatment as prevention for hepatitis $\mathrm{C}$ : Meeting the needs of people who inject drugs? International Journal of Drug Policy. 2015;26(10):963-9.

9. Falade-Nwulia O, Sulkowski MS, Merkow A, Latkin C, Mehta SH. Understanding and addressing hepatitis $C$ reinfection in the oral direct-acting antiviral era. Journal of viral hepatitis. 2018;25(3):2207.

10. Springer SA. Hepatitis $C$ virus reinfection rate among persons who use drugs and are maintained on medication treatment for opioid use disorder. Clinical infectious diseases : an official publication of the Infectious Diseases Society of America. 2019.

11. Grebely J, Hajarizadeh B, Lazarus JV, Bruneau J, Treloar C. Elimination of hepatitis C virus infection among people who use drugs: Ensuring equitable access to prevention, treatment, and care for all. The International journal on drug policy. 2019;72:1-10.

12. Falade-Nwulia $\mathrm{O}$, Sulkowski M. The HCV care continuum does not end with cure: A call to arms for the prevention of reinfection. Journal of hepatology. 2017;66(2):267-9. 
13. Hagan H, Pouget ER, Des Jarlais DC. A systematic review and meta-analysis of interventions to prevent hepatitis $C$ virus infection in people who inject drugs. The Journal of infectious diseases. 2011;204(1):74-83.

14. Martin NK, Skaathun B, Vickerman P, Stuart D. Modeling Combination HCV Prevention among HIVinfected Men Who Have Sex With Men and People Who Inject Drugs. AIDS reviews. 2017;19(2):97104.

15. Heimer R, Clair S, Grau LE, Bluthenthal RN, Marshall PA, Singer M. Hepatitis-associated knowledge is low and risks are high among HIV-aware injection drug users in three US cities. Addiction (Abingdon, England). 2002;97(10):1277-87.

16. Koester S, Heimer R, Barón AE, Glanz J, Teng W. RE:"Risk of Hepatitis C virus among young adult injection drug users who share injection equipment". American journal of epidemiology. 2003;157(4):376-.

17. Paintsil E, Binka $M$, Patel $A$, Lindenbach $B D$, Heimer R. Hepatitis $C$ virus maintains infectivity for weeks after drying on inanimate surfaces at room temperature: implications for risks of transmission. The Journal of infectious diseases. 2014;209(8):1205-11.

18. Paintsil E, He H, Peters C, Lindenbach BD, Heimer R. Survival of hepatitis C virus in syringes: implication for transmission among injection drug users. The Journal of infectious diseases. 2010;202(7):984-90.

19. Grau LE, Green TC, Singer M, Bluthenthal RN, Marshall PA, Heimer R. Getting the message straight: effects of a brief hepatitis prevention intervention among injection drug users. Harm reduction journal. 2009;6(1):36.

20. Grau LE, Bluthenthal RN, Marshall P, Singer M, Heimer R. Psychosocial and behavioral differences among drug injectors who use and do not use syringe exchange programs. AIDS and behavior. 2005;9(4):495-504.

21. Fisher JD, Fisher WA. Changing AIDS-risk behavior. Psychological bulletin. 1992;111(3):455-74.

22. Chang SJ, Choi S, Kim S-A, Song M. Intervention strategies based on information-motivationbehavioral skills model for health behavior change: a systematic review. Asian Nursing Research. 2014;8(3):172-81.

23. Butner JL, Gupta N, Fabian C, Henry S, Shi JM, Tetrault JM. Onsite treatment of HCV infection with direct acting antivirals within an opioid treatment program. Journal of substance abuse treatment. 2017;75:49-53.

24. Severe B, Tetrault JM, Madden L, Heimer R. Co-Located hepatitis C virus infection treatment within an opioid treatment program promotes opioid agonist treatment retention. Drug and alcohol dependence. 2020;213:108116.

25. Rounsaville BJ, Carroll KM, Onken LS. A stage model of behavioral therapies research: getting started and moving on from Stage I. Clinical Psychology: Science and Practice. 2001;8(2):133-42.

26. Bandura A, Ross D, Ross SA. Vicarious reinforcement and imitative learning. The Journal of Abnormal and Social Psychology. 1963;67(6):601-7. 
27. Bandura A. Social foundations of thought and action. Englewood Cliffs, NJ. 1986;1986:23-8.

28. Bowman S, Grau LE, Singer M, Scott G, Heimer R. Factors associated with hepatitis B vaccine series completion in a randomized trial for injection drug users reached through syringe exchange programs in three US cities. BMC public health. 2014;14(1):820.

29. Heimer R, Grau LE, Singer M, Scott G, Marshall PA, Hu Y, et al. Hepatitis B virus prevalence and vaccination rates among Hispanic injection drug users participating in a vaccination campaign. Journal of drug issues. 2008;38(1):335-50.

30. Chicago Recovery Alliance. Better Vein Care/Safer Injection Guide 2018 [Available from: https://anypositivechange.org/better-vein-care/.

31. Canadian AIDS Treatment Information Exchange [CATIE]. Sharp Shooters 2020 [Available from: http://librarypdf.catie.ca/ATI-70000s/70095.pdf.

32. Latkin CA, Sherman S, Knowlton A. HIV prevention among drug users: outcome of a network-oriented peer outreach intervention. Health psychology : official journal of the Division of Health Psychology, American Psychological Association. 2003;22(4):332-9.

33. Heimer R, Barbour R, Palacios WR, Nichols LG, Grau LE. Associations between injection risk and community disadvantage among suburban injection drug users in southwestern Connecticut, USA. AIDS and behavior. 2014;18(3):452-63.

34. Levesque CS, Williams GC, Elliot D, Pickering MA, Bodenhamer B, Finley PJ. Validating the theoretical structure of the Treatment Self-Regulation Questionnaire (TSRQ) across three different health behaviors. Health Educ Res. 2007;22(5):691-702.

35. Williams G, Ryan R, Deci E. Health-care, self-determination theory packet. Health-care, selfdetermination theory questionaire. 2019.

36. Khatmi N, Dany L, Ndiaye K, Carrieri P, Rojas Castro D, Roux P. Impact of an educational intervention on risks associated with drug injection, and on psychosocial factors (PSF) involved in initiating and maintaining new health behaviors over time. Addictive behaviors. 2018;87:222-30.

37. Marotta PL, Terlikbayeva A, Gilbert L, Hunt T, Mandavia A, Wu E, et al. Intimate relationships and patterns of drug and sexual risk behaviors among people who inject drugs in Kazakhstan: A latent class analysis. Drug and alcohol dependence. 2018;192:294-302.

38. Latka MH, Hagan H, Kapadia F, Golub ET, Bonner S, Campbell JV, et al. A randomized intervention trial to reduce the lending of used injection equipment among injection drug users infected with hepatitis C. Am J Public Health. 2008;98(5):853-61.

\section{Figures}




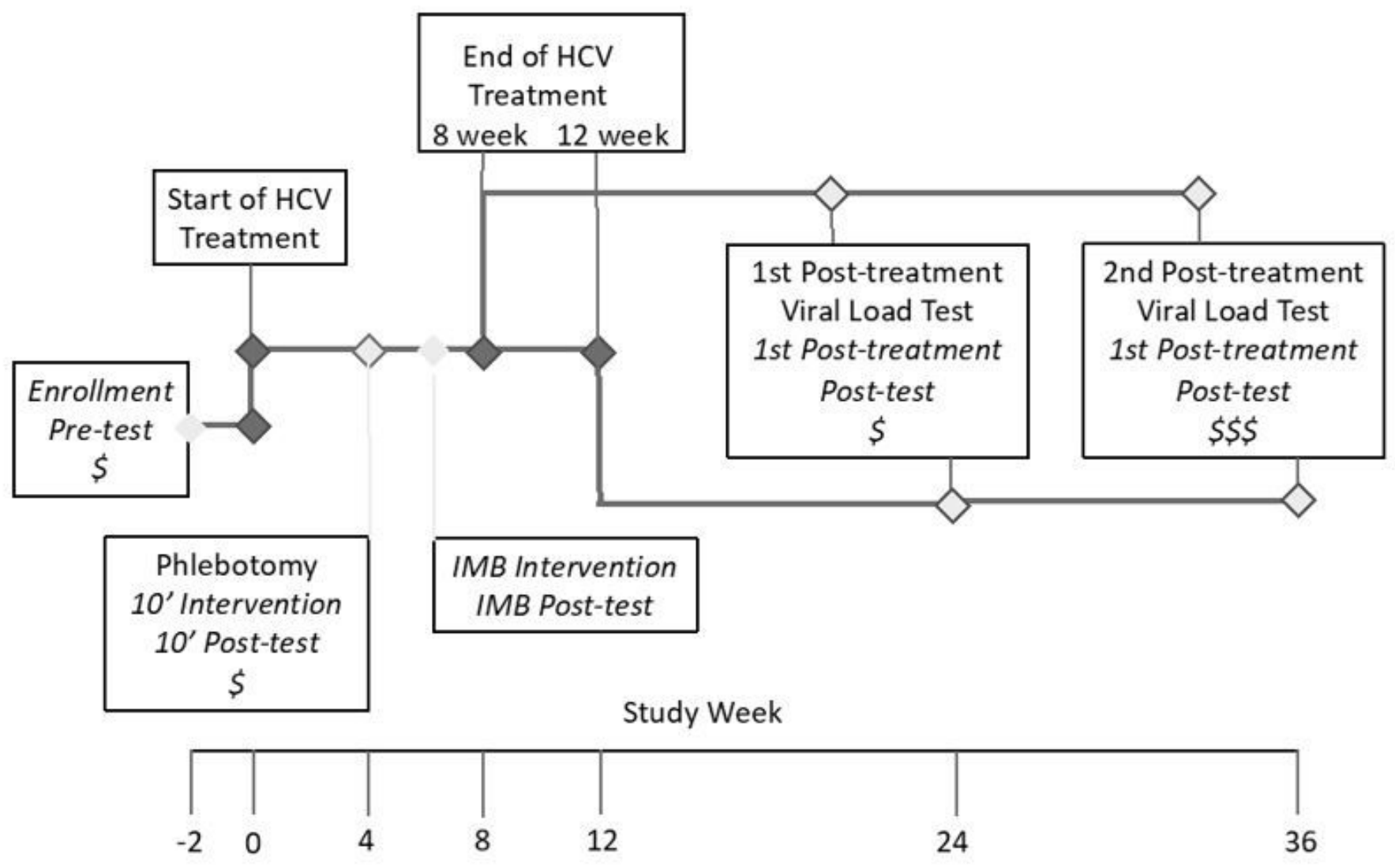

Figure 1

Intervention and Data Collection Schematic. Times are depicted for intervention only activities (solid squares), treatment only events (open diamonds), and intervention activities concurrent with treatment events (closed diamond). Schematic accounts for different lengths of DAA treatment. 